http://jmscr.igmpublication.org/home/ ISSN (e)-2347-176x ISSN (p) 2455-0450

crossref DOI: https://dx.doi.org/10.18535/jmscr/v8i2.129

\title{
Dournal Of Medical Science And Clinical Research

\section{Clinical Profile and Neurodevelopmental Outcome of Neonatal Sepsis in a Tertiary Care Children Hospital from Eastern India}

Authors

\section{Dr Sreejata Mazumdar ${ }^{1}$, Dr Suprit Basu ${ }^{2 *}$, Dr Mala Bhattacharya ${ }^{3}$}

${ }^{1}$ Senior Resident, Pediatric Medicine, South Howrah State General Hospital, Howrah, West Bengal

${ }^{2}$ Assistant Professor, Pediatric Medicine, Institute of Post Graduate and Medical Education Research, Kolkata, West Bengal

${ }^{3}$ Professor, Pediatric Medicine, Jagannath Gupta Institute of Medical Sciences Kolkata West Bengal *Corresponding Author

Dr Suprit Basu

\begin{abstract}
Objective: Study the profile of neonatal sepsis, follow up the patients with neonatal sepsis in high risk clinic for lyear to assess neurodevelopmental and other physical morbidities \& intervene the issues at earliest possibility.

Materials and Methods: Prospective, observational, longitudinal study done in high risk clinic for 1year in a tertiary care hospital of eastern India.

Result: Major (34.3\%) causative organism of neonatal sepsis is Klebsiella.18.18\% of total study population had neurodevelopmental delay. Growth failure, microcephaly, repeated hospital admission is more common in babies with neurodevelopmental delay. Persistent tone abnormality, abnormal electrophysiological are commonly associated with delayed neurodevelopment.

Conclusion: In our study we found that neurodevelopmental delay is common in neonatal sepsis. Regular follow up with appropriate screening test can identify it early \& early stimulation can prevent or reduce developmental delay.

Keywords: Neonatal sepsis, neurodvelopmental outcome, follow up.
\end{abstract}

\section{Introduction}

With improving neonatal care services the survival rate of patients with neonatal sepsis are gradually increasing, specially by using appropriate antibiotics, devices like continuous positive airway pressure and mechanical ventilation. Despite substantial decrease in neonatal mortality there is scope of improvement in incidence of morbidities and adverse neurodevelopmental outcomes among the survivors .This emphasizes the need to look for outcome and assessment of these patients for proper evaluation and appropriate therapy.

\section{Materials and Methods}

A. Study Area- High Risk Clinic of Department of Pediatric Medicine, Dr. B.C Roy PGIPS

B. Study Population- Neonates having neonatal sepsis

C. Study Period- June 2016 - May 2017

D. Sample Size - 220 
E. Sample Design- neonates having blood culture positive and sepsis screen positive neonatal sepsis \& were followed up at High Risk Clinic up to 1year of age.

F. Inclusion Criteria- The following high risk infants were enrolled in the study after their discharge -
1) Neonates having blood culture positive sepsis
2) Neonates having sepsis screen positive sepsis

\section{G. Exclusion Criteria}

1) Infants with major congenital anomaly

2) Death before discharge

3) Parents those who are not willing to follow up

4) Babies with severe birth asphyxia (HIE II \& III)

5) Babies with bilirubin encephalopathy

6) Babies with extreme prematurity

H. Study Design- Prospective,

Observational, Longitudinal study.

\section{The Follow up Schedule}

\begin{tabular}{|c|l|}
\hline Cohort & Follow up schedule \\
\hline $\begin{array}{l}\text { 1. Infants with } \\
\text { birth weight }< \\
1800 \text { gm and } \\
\text { or gestational } \\
\text { age }<35 \text { wks } \\
\text { the baby has adjusted well in home } \\
\text { environment. Thereafter they were } \\
\text { checked every 2 weeks until a } \\
\text { weight of } 3 \mathrm{~kg} \text { is achieved. They } \\
\text { were also seen at 1,3,6,9,12, } \\
\text { months of corrected age. }\end{array}$ \\
\hline $\begin{array}{l}\text { Other } \\
\text { conditions }\end{array}$ & At $1,3,6,9,12$ months of age \\
\hline
\end{tabular}

This is the minimum number of visits of high risk neonates. For those babies who had ongoing illnesses, we stressed upon more frequent visits as per individual need.

\section{Result and Analysis}

251 patients with neonatal sepsis were enrolled for the study.5 patients died during follow up and 26 patients did not come for regular follow up. so, the study includes 220 patients with neonatal sepsis who were followed up for 1 year in high risk clinic during the period of June 2016 to May 2017.

Out of 220 babies 97 babies were female, 123 were male \& 49 were preterm, 171 were term, 49 babies were small for gestational age (SGA),171 were appropriate for gestational age (AGA)

Out of 220 patients $93(42.3 \%)$ patients had early onset neonatal sepsis (EOS) and 127(57.7\%) patients had late onset neonatal sepsis (LOS). In our institution all the patients are out born. This may be the reason behind majority of patients having LOS.

Out of 220 neonates $67(30.5 \%)$ patients had blood c/s positive sepsis and $153(69.5 \%)$ patients had sepsis screen positive sepsis. Among 49 preterm babies $14(28.6 \%)$ babies \& out of 171 term neonates $53(31 \%)$ had blood c/s positive sepsis.

Among 67 blood c/s +ve sepsis cases 25(37.3\%) patients had meningitis \& among 153 sepsis screen +ve cases,23(15\%) babies were diagnosed to have meningitis. It means babies with blood c/s positive sepsis have significantly higher chance of meningitis. $(52.1 \%, 25$ out of 48 patients with meningitis). Among the 49 preterm babies $10(20.4 \%) \&$ out of 171 term babies 38(22.2\%) babies had meningitis.

Among 67 blood c/s +ve sepsis cases, majority of the sepsis were caused by Klebsiella pneumonia (23patients,34.3\%) followed by E.coli (14.9\%), Staphylococcus aureus (11.9\%),Acinatobactor baumani (10.4\%), Pseudomonus aeruginosa (7.5\%), coagulase -ve staphylococcus (CONS, 7.5\%), Sphingomonus

paucimobilis $(4.5 \%)$, Candida albicans $(4.5 \%)$, enterococcus (3\%), micrococcus (1.5\%). Klebsiella is the main causative organism, $40 \%$ \& $29.7 \%$ respectively in EOS \& LOS, followed by Staphylococcus aureus, CONS, Pseudomonas, Acinetobactor (all 10\%) in EOS, in LOS Among 48 patients with meningitis $25(52.1 \%)$ patients had blood c/s positive. Klebsiella was the main causative organism $(56 \%)$ of blood c/s +ve sepsis with meningitis. Rest of the organisms were Staphylococcus (32\%), E.coli, Acinatobactor, Pseudomonas $4 \%$ each. 
Among 220 infants, 40 infants $(18.18 \%)$ had neurodevelopmental delay as confirmed by Developmental assessment scale for Indian infant (DASII) test.In initial Denver developmental screening test (DDST) done at 6 months of age 58 patients had caution/delay. All the patients were treated with early stimulation and physiotherapy. Subsequenty 19 babies (32.8\%) attained age appropriate milestones, but 39 infants $(67.2 \%)$ had persistent developmental delay confirmed by DASII. Thus it shows babies having delay in initial DDST significantly have higher chance of neurodevelopmental delay in future.

Among the 40 patients, having mental developmental delay majority had moderate delay (47.5\%), followed by severe delay in $35 \%$ babies and mild delay in $17.5 \%$ babies. Among the 40 patients with motor developmental delay majority had moderate delay(55\%), followed by severe delay in $42.5 \%$ babies and mild delay in $2.5 \%$ babies.

Among the 40 patients having delayed neurodevelopment $32(80 \%)$ patients $\&$ among the 180 patients having normal neurodevelopment $58(32.25 \%)$ patients had weight less than $3^{\text {rd }}$ percentile at 1year of age. $30 \%$ among babies with neurodevelopmental delay as compared to $10 \%$ among babies with normal neurodevelopment failed to gain expected length at 1 year of age.

Out of 220 high risk neonates 37.4\% AGA babies (64 out of 171) and 53.1\% SGA babies(26 out of 49) had growth failure in the form of weight less than $3^{\text {rd }}$ percentile at $1 \mathrm{yr}$ of age. Failure to gain expected length at 1year of age was slightly higher in SGA babies (14.3\%) than AGA (13.5\%) babies as well as in preterm (16.3\%) than term (12.9\%) babies. Among the 40 babies having developmental delay $82.5 \%$ (33 out of 40) babies had microcephaly and out of 180 normal babies $30 \%$ (54 out of 180) had microcephaly.

Out of 220 subjects,60 (27.3\%) had abnormal neurologic examination at discharge. But among them, only 30 infants $(50 \%)$ exhibited neurodevelopmental delay at $1 \mathrm{yr}$ of age. Among the 160 patients with normal neurological examination at discharge $10(6.3 \%)$ babies had developmental delay.

Neonates with blood c/s positive sepsis with meningitis had maximum number of delay (37.5\%, 15 patients out of 40).The neonates having sepsis screen positive sepsis without meningitis had minimum number of delay( $12.5 \%, 5$ babies out of 40$)$.

In the initial visit, 110 out of 220 infants had abnormality in muscle tone. However on continued follow up, 67 infants normalized (transient tone abnormality or TTA) and rest 43 infants had persistent tone abnormality (PTA) till 1year of age (35 patients had hypertonia, 8 patients had hypotonia). 34(97.1\%) patients had delayed neurodevelopment among 35 patients having persistent hypertonia.6 patients $(75 \%)$ had developmental delay among 8 patients having persistent hypotonia. All the 67 patients having transient tone abnormality had normal developmental outcome. Among the 67 patients having blood c/s +ve sepsis 25 babies(37.3\%) had hypertonia,3babies $(4.5 \%)$ had hypotonia, 27 babies $(40.3 \%)$ had transient tone abnormality which normalized during follow up. Among 153 patients having sepsis screen positive sepsis 10 babies $(6.5 \%)$ had hypertonia,5 babies $(3.3 \%)$ had hypotonia and 40 babies had transient tone abnormality. Among 48 patients having meningitis 20 babies $(41.7 \%)$ had persistent hypertonia, 4 babies (8.3\%) had persistent hypotonia, and $15(31.3 \%)$ patients had transient tone abnormality. Among 172 patients having sepsis screen positive sepsis 15 babies (8.2\%) had hypertonia, 4 babies $(3.3 \%)$ had hypotonia and $52(30.2 \%)$ babies had transient tone abnormality.

Among 40 patients with delayed neurodevelopment 25 patients (62.5\%) had abnormal Brainstem evoked response audiometry (BERA), whereas out of 180 patients with normal neurodevelopment 4 patients $(2.2 \%)$ had abnormal BERA. Thus it shows that patients having abnormal BERA have significantly higher chance of neurodevelopmental delay. 
Among the 38 patients having abnormal cranial ultrasound at discharge, 28 patients $(73.7 \%)$ had neurodevelopmental delay,10 babies(26.3\%) had no delay. Out of 182 patients having normal neuroimaging at discharge only 12 babies $(6.6 \%)$ had neurodevelopmental delay.

Electro encephalogram (EEG) done in all the high risk babies with or without convulsion.It is shown that total 23 patients had abnormal EEG.out of them 18 patients $(78.3 \%)$ had developmental delay. Only 22 babies (11.2\%) out of 197 patients with normal EEG had developmental delay. So, it is seen that abnormal EEG is significantly associated with developmental delay.

In this study out of 220 high risk neonates 24 patients developed seizure disorder during 1 year follow up. Out of them 13 patients (54.2\%) had abnormal EEG, whereas out of 197 patients with normal EEG,11 patients(5.6\%) developed seizure disorder. Thus, it means abnormal EEG is significantly associated with seizure disorder.

All high risk neonates had undergone fundoscopy \& Visual evoked potential (VEP) during follow up.15 patients had pale retina with abnormal VEP $\& 14$ of them had delayed neurodevelopmental outcome.4 patients had b/l convergent squint.

Among 40 patients with developmental delay 14 patients $(35 \%)$ had abnormal eye examination finding. Out of 180 patients with normal development only 4 patients $(2.2 \%)$ had abnormal eye examination. This means developmental delay is significantly associated with abnormal eye examination.

$57.5 \%$ patients with developmental delay had one or more readmissions during 1 year follow up. While only $8.9 \%$ patients with normal development were readmitted during follow up. Thus it shows that developmental delay is significantly associated with repeated hospital admission.

\section{Discussion}

The very purpose of setting up expensive modern, dedicated NICU would be defeated if there are no follow up services for NICU graduates. Hence this study was designed to present overview of the results seen while following up high risk neonates at our high risk clinic.

Due to many reasons, some infants are not brought for regular follow up. In our study, the dropout rate was $10.3 \%$ which is comparable to the $7.7 \%$ drop out rate mentioned in the study by Chaudhari $\mathrm{S}$ et $\mathrm{al}^{1}$ published in Indian Pediatrics 2009.

In our study $42.3 \%$ had early onset sepsis and $57.7 \%$ had late onset sepsis, as compared to Mamatha $\mathrm{P}$ et $\mathrm{al}^{2}$ (53.6\% EOS and $46.4 \%$ LOS).

In our study 67 out of 220 (30.4\%) neonates had blood culture positive sepsis. It is comparable to study done by Mamatha $\mathrm{P}$ et al \& V.L Jayasimha et al, 2017, which had $21.7 \% \& 44.4 \%$ blood culture positive sepsis respectively.

In our study 48 out of 220 neonates had meningitis $(21.8 \%)$.whereas blood culture positive was found in $52.1 \%$ meningitis patients. It is comparable to other studies. Laving et al. ${ }^{3}$ Anjos De Silva et al. (2007) and Tisukumara et al.(2009) ${ }^{4}$ reported prevalence of meningitis in neonates with late onset sepsis as $17.9 \%, 17 \%$ and $17.2 \%$ respectively.

In our study majority of the blood c/s positive sepsis was due to Klebsiella pneumonia (34.3\%) as compared to study done by V.L Jayasima et al(2017).In this study, the most frequent isolate was Klebsiella pneumoniae $(35.4 \%)$ in both EOS and LOS.This was in accordance with other Indian studies NNPD 2003, Kayange et $\mathrm{al}^{5}$, Kumar et $\mathrm{al}^{6}$, Roy et $\mathrm{al}^{7}$. Staphylococcus aureus was the commonest Gram positive organism and was second most common organism among all isolates as found in our study.

In our study majority of the sepsis meningitis was caused by Klebsiella pneumonia(56\%), followed by Staphyococcus aureus (32\%), as also study done by Roshi et $\mathrm{al}^{8}$, Meningitis was seen in $60.7 \%$ cases of proven gram negative sepsis and $30.7 \%$ cases of proven gram positive sepsis.

Assessment of growth upto 1 year of age revealed that growth failure in respect to weight, height and length was significantly greater among infants 
with neurodevelopmental delay. This may be due to the presence of feeding problems and increased incidence of intercurrent illness among these infants.

Although the incidence of growth failure was higher among preterm and SGA infants compared to full term and AGA infants respectively, the differences were not statistically significant.62.6\% of AGA infants maintained their normal growth rate in extrauterine life in our study. This result was comparable to the study by Maribel Campos et al where $42.11 \%$ of AGA infants maintained adequate growth rate. However catch up growth was achieved by only $42.6 \%$ of SGA infants in our study as compared to study conducted by Maribel C et $\mathrm{al}^{16}$, where $42 \%$ of SGA infants achieved catch up growth.

In their study, Maureen Hack et al ${ }^{88}$ described that more SGA infants (25\%) than AGA infants (14\%) had subnormal head circumference on follow up, indicating failure to catch up in head growth among SGA infants. Although in our study, incidence of microcephaly was higher among SGA infants (46.9\%) than AGA infants (37.4\%) the difference was not statistically significant. In our study microcephaly significantly found in patients with developmental delay $(82.5 \%)$ as compared to normal development (30\%).15\% of total high risk cohort had microcephaly as compared to study by Klinger et $\mathrm{al}^{9}$ where $25 \%$ of patients had microcephaly at the age of one year.

Our study revealed that infants with abnormal neurologic examination at discharge had significantly higher incidence of developmental delay. The sensitivity, specificity, positive predictive value (PPV) and negative predictive value (NPV) of abnormal neurologic examination was $50 \%, 93.8 \%, 75 \% 83.3 \%$ respectively $(71 \%$, $96 \%, 92 \%, 82 \%$ respectively in the study by Thompson CM et $\mathrm{al}^{15}$ ).

The Amiel Tison Method ${ }^{12}$ of tone assessment revealed that the prevalence of Transient Tone Abnormality (TTA) among high risk infants was $30.5 \%$ (35.2\% in the study by Chaudhuri $\mathrm{S}$ et $\left.\mathrm{al}^{14}\right)$.There was no significant difference in the proportion of TTA between full terms and preterms $(27.9 \%$ and $40.78 \%$ respectively); between AGA and SGA infants $(36.7 \%$ and $28.7 \%$ respectively) - the results were almost similar to the findings of Chaudhuri $\mathrm{S}$ et $\mathrm{al}^{13}$, where the prevalence of TTA among full terms, preterms, AGA, SGA infants were $30.8 \%, 35.9 \%$, $37.8 \%, 36.4 \%$ respectively. Among those infants with Persistent Tone Abnormality (PTA),81.3\% were hypertonic, $18.6 \%$ were hypotonic. In our study $50 \%$ patients with sepsis+meningitis had persistent tone abnormality as compared to study done by Nitin $\mathrm{S}$ et al $(48.2 \%)^{10}$

In DASII test, $40(18.18 \%)$ of the 220 infants were confirmed to have developmental delay. This finding is slightly higher when compared to the Asia Pacific Childhood Disability Update $2005^{15}$, where the incidence of developmental delay in NICU survivors were $10-20 \%$. This was possibly due to the fact that infants with normal development dropped out from the study, whereas infants with developmental delay regularly attended the High Risk Clinic. However, no statistically significant difference was noted in the incidence of developmental delay among full terms, preterms, and among AGA and SGA infants, as also described by Maribel Campos et $\mathrm{al}^{16}$ in their study. In our study blood c/s positive with meningitis patients were the major group $(37.5 \%)$ to have moderate to severe developmental delay, as compared to $45 \%$ moderate to severe delay in study done by Nitin $\mathrm{S}$ et $\mathrm{al}^{10}$.

Out of 58 infants who were rated 'Caution' in DDST test, 19 infants i.e. $32.8 \%$ had normal development during one year follow up after Early Stimulation was prescribed. Hence, Early Stimulation is a very effective measure to promote normal development in these infants who were progressing towards developmental delay.

In our study abnormal BERA found in $28.4 \%$ blood c/s positive sepsis and $37.5 \%$ patients having meningitis, which is significantly higher than patients with blood $\mathrm{c} / \mathrm{s}$ negative without meningitis. This finding was significantly higher 
as compared to study of Klinger et a ${ }^{9} \&$ Stevens et $\mathrm{al}^{11}$ where hearing loss found in $8.5 \%$ \& $3.6 \%$ patients with meningitis respectively. Incidence of BERA abnormality was significantly higher among infants with developmental delay $(62.5 \%$, $\mathrm{p}<0.0001)$. Similar findings was also described by Mukhopadhyay K in their study ${ }^{98}(\mathrm{p}=0.004)$.

Infants with abnormality in CUS (cranial ultrasound) at discharge were found to have significantly higher incidence of developmental delay $(\mathrm{p}<0.0001)$. Out of those 38 infants who had abnormal CUS at discharge, 10(26.3\%) infants had normal neuroimaging findings on follow up \& all of them had normal outcome. Thus it shows 10 infants, despite having abnormalities in neuroimaging, had normal developmental outcome- this could be attributed to the neuroplasticity of the developing brain. We also found that babies with sepsis+meningitis had more $(48 \%)$ abnormal neuroimaging than patients without meningitis (7.7\%).

EEG abnormality was found to be significantly higher in cases of infants with delay ( $p$ $<0.0001)$. Sensitivity, specificity, PPV, NPV of abnormal EEG was found to be $45 \%$, $97.2 \%, 78.3 \%, 88.8 \%$ respectively. In 2001,in European Journal of Pediatric Neurology, Znistra $E$ et $\mathrm{al}^{19}$ described that a single EEG study, particularly during acute phase of disease, may suggest a more ominous outcome, but serial EEG can be better in predicting outcome. This aspect was also demonstrated in our study- out of 23 infants with abnormal EEG, serial EEG monitoring showed normalization in cases of 6 infants (who had normal outcome), while 17 infants had persistent abnormality. All these 17 infants had developmental delay.

Abnormal eye examination was significantly associated with developmental delay ( $p$ value $<0.0001$ ) in our study, similar finding was found in Stevens et $\mathrm{al}^{11}$ where $17 \%$ patients with developmental delay had abnormal eye examination.

Readmission rate was found to be significantly higher among delayed infants $(\mathrm{p}<0.0005)$.
Recurrent seizure \& LRTI were the most common morbidity and the most common cause of hospitalization. This finding was also described by Doyle LW et $\mathrm{al}^{20}$ in their study published in 2003. Other causes of admission were seizures, anemia requiring transfusion and need for surgeries.

In our study $10.9 \%$ patients had seizure disorder requiring anticonvulsant compared to $15 \%$ patients with seizure disorder in study by Stevens et al. ${ }^{11}$

Thus, majority of the findings of our study correlated well with many National and International studies. Few differences were due to short (1 year) period of follow up and smaller number of subjects in each high risk groups compared to the quoted studies.

\section{Conclusion}

Klebsiella is the main causative organism of neonatal sepsis in this part of the country. Neonatal sepsis with meningitis can cause neurodevelopmental delay. Here lies the regular follow up of neonates in high risk clinic. Timely follow up with appropriate screening test can identify developmental delay early \& early stimulation can prevent or reduce developmental delay to large extent.

No conflict of interest

\section{Bibliography}

1. Chaudhari S, Deo B. Neurodevelopmental assessment in the first year with emphasis on evolution of tone. Indian Pediatrics 2006;43:527-534.

2. Mamatha P Samaga and B. Sumangala. Bacteriological profile of neonatal Septicaemia in MIMS, Mandya, India. Int.J.Curr.Microbiol.App.Sci (2016) 5(3): 495-501

3. Laving AM, Musoke RN, Wasunna AO, Revathi G. Neonatal bacterial meningitis at the new-born unit of Kenyatta national hospital. East Afr Med J. 2003 Sep;80(9):456-62. 
4. Tiskumara R, Fakharee $\mathrm{SH}$, Liu CQ, Nuntnarumit P, Lui KM, Hammoud M, et al. Neonatal infections in Asia. Arch Dis Child Fetal Neonatal Ed. 2009 Mar;94(2):F144-8.

5. Kumar GD, Ramachandran VG, Gupta P. Bacteriological analysis of blood culture isolates from neonates in a tertiary care hospital in India. J Health Popul Nutr 2002; 20(4):343-34

6. Kayange N, Kamugisha E, Mwizamholya DL, Jeremiah S, Meshana SE. Predictors of positive blood culture and deaths among neonates with suspected neonatal sepsis in a tertiary hospital, Mwanza-Tanzania. BMC Pediatrics 2010; 10:39.

7. Roy I, Jain A, Kumar M, Agarwal SK. Bacteriology of neo- natal septicemia in a tertiary care hospital of Northern In- dia. Indian Journal of Medical Microbiology 2002; 20 (3):156-159

8. Bhagat $\mathrm{R}$ et al.Incidence of meningitis in ate onset sepsis. Int $\mathbf{J}$ Contemp Pediatr. 2015 May;2(2):96-102.

9. Klinger G, Chin Cn, Beyene J, Perlman M. Predicting the outcome of neonatal bacterial meningitis. Pediatrics. 2000;106:477-482.

10. Nitin S. Mehkarkar, P. S. Patil , Retrospective assessment neurodevelopmental outcome of neonatal meningitis. International Journal of Recent Trends in Science And Technology, ISSN 2277-2812 E-ISSN 2249-8109, Volume 7, Issue 3, 2013

11. Stevens JP, Eames M, Kent A, Halket S, Holt D, Harvey. Long term outcome of neonatal meningitis. Arch Dis Child 2002;F179-F184.

12. Amiel Tison C. A method for neurologic evaluation within the first year of life. Curr Prob Pediatr 1976; 7(1): 1-50
13. Kubota $\mathrm{T}$ et al. Combination of neonatal ultrasonography and electroencephalography- sensitive means of early diagnosis of periventricular leucomalacia. Brain Dev 2002; 24: 698702

14. Chaudhari $S$ et al. Transient tone abnormalities and cognitive outcome at five years. Indian Pediatr. Nov 17 2010; 47:931-935.

15. Cerebral Palsy- Follow up of High Risk Neonates (Asia Pacific Childhood Disability Update 2005 ). Sarojini Budden MD, FRCP: Oregon Health and Sciences University and Pediatric Development Program Neonatal Follow up Clinic Legacy. Emanuel Children Hospital, Portland, Oregon. www.pediatriconcall.com/FORDOCT.

16. Campos $\mathrm{M}$ et al. Comparison of post discharge growth in AGA and SGA infants. Ethnicity and Disease. Spring 2008; Vol. 18

17. Mauren Hack et al. Differential effect of intrauterine and postnatal brain growth failure in infants of very low birth weight. Am J Dis Child 1989; 143(1): 63-68

18. Bhagat $\mathrm{R}$ et al. Int $\mathrm{J}$ Contemp Pediatr. 2015 May;2(2):96-102

19. Hussain $\mathrm{N}$ et al. The current incidence of ROP. Pediatrics 1999; Vol104, No. 3:e26.

20. Doyle LW et al. Health and hospitalization after discharge in high risk infants. Semim Neonatol 2003 Apr 8(2): 137-145. 